\title{
Analysis of Hydromagnetic Double Exothermic Chemical Reactive Flow with Convective Cooling through a Porous Medium under Bimolecular Kinetics
}

\author{
F. O. Akinpelu, R. A. Oderinu, A. D. Ohaegbue* \\ Department of Mathematics, Ladoke Akintola University of Technology, Ogbomoso, Nigeria
}

\begin{abstract}
In this study, the analytical solution of steady hydromagnetic double exothermic combustible reaction fluid flow in a porous medium with convective cooling wall is presented. The viscous heating reactive liquid is totaling exothermic without consumption of material. The combustion reaction of the fluid takes place in a Poiseuille device, and it is been propelled by pressure gradient and pre-exponential bimolecular kinetics. The device is exposed to convective cooling to keep the reactive hydromagnetic fluid from distortion. The weighted residual method (WRM) is analytically used to get the numerical values for the dimensionless nonlinear governing equations. The solution to temperature and velocity distribution is carried out and the result is graphically depicted. The Nusselt number and skin friction coefficient is also showed for some significant parameters engrained in the flow and the solution obtained is compared with numerical method. As obtained in the study, the second exothermic reaction term increases the combustion process; hence the term will assist in reducing toxic discharge from the engines that pollute the environment. The Frank-Kamenetskii parameter contributes highly to system thermo-fluid destruction; as such it must be monitored.
\end{abstract}

DOI:10.46481/jnsps.2022.525

Keywords: Exothermic reaction, Convective cooling, Bimolecular kinetics, Porous medium

Article History :

Received: 19 December 2021

Received in revised form: 06 February 2022

Accepted for publication: 19 February 2022

Published: 28 February 2022

(c)2022 Journal of the Nigerian Society of Physical Sciences. All rights reserved. Communicated by: J. Ndam

\section{Introduction}

The idea of laminar flow of fluid particles along a medium continues to receive tremendously attention because of its application to fluid mechanics particularly in industries. Its significant has been influenced by many engineering analyst, biological science, atomic control, aerodynamics of plasma and magnetohydrodynamic (MHD) system, Ellahiand Afzal [1]. The flow of exothermic reactive fluid is useful in industrial and engi-

${ }^{*}$ Corresponding author tel. no: +2348061198971

Email address: ohaegbueanthony@gmail.com (A. D. Ohaegbue ) neering system and is frequently accompanied with heat transfer which has long been called combustion, Makinde [2]. Some convective fluids flows are caused by the generating or absorbing of heat which results to a fluid chemical reaction. Heat source encourages the distribution of heat which then changes the amount of particles deposition within the arrangements such as electric clip, nuclear reactor, semiconductor etc. Generation or the absorption of heat has been assumed to depend on temperature. The effect of heat production or absorption of nonhomogenous state in a micro polar fluid with variable conductivity was considered by Chen [3]. The results show the dependent generation of heat at the surface is less to temperature 
dependent generation. The authors $[4,5]$ studied the impact of non-uniform heat supply on hydromagnetic micro polar fluid with radiative in permeable media.

Many studies have been carried out on exothermic chemical reactive fluid flows and its applicability in energy storage systems, atomic reactor plan, geophysics, polymer expulsion and MHD reactive fluid flow. Consequent to its applications, Gbadeyan and Hassan [6] worked on the reaction of Couette variable viscosity fluid flow for Arrhenius case. Transfer of exothermic heat flow through a pipe was investigated by Marcello et al. [7] under different chemical kinetics, Adomian decomposition was used to solve the problem. The comprehensive survey on generation of heat by hydromagnetic fluid flow considering all the basic physical properties was reported in [8-9]. Among the numerous suggested models is a single step exothermic reaction while some are under convective cooling. Hazarika et al. [10] worked on the relevance of variable properties on MHD flow through a perpendicular plate. They declared that the velocity outline rises and the parameter of heat conductivity decreases. Dulal \& Hiranmoy [11] analyzed the effect of heat on variable heat conductivity and MHD of non-Darcy mixed convective flow of concentration over an extending sheet. The impact of MHD on heat distribution over an extented walls fixed in porous medium with heat source, variable viscousity and viscous dissipation was discussed by Hunegnaw \& Naikoti [12]. Hassan and Maritz [13] investigated the chemical reaction on hydromagnetic flow in stationary parallel convective cooling walls. The result shows that a rise in magnetic intensity and porous medium values result to a fall in the velocity profiles and a rise in the values of porous medium, activation energy and magnetic force with convective cooling terms decreases the temperature of the fluid. Dulal [14] examined temperature and mass transfer in a fixed flow of viscous fluid on a distending perpendicular sheet, taking buoyancy and heat radiation into consideration. Hazarika and Utpal [15] investigated the effect of energy conductivity and variable properties on hydromagnetic flow across a perpendicular plate. They observed that when the heat conductivity value is reduced, the velocity distribution rises. Adesanya and Folade [16] investigated the flow of hydromagnetic material through a porous media at the third grade level. Bala and Suneetha [17] investigated the effect of homogeneous and heterogeneous chemical reactive stagnation flow on the MHD of a micropolar fluid through a permeability shrinking/stretching surface plate fixed in a porous wall. Salawu [18] reported on the heat transfer on third grade combustive echemical reactive flow and absorption of heat with convective cooling in a porous medium. Seth et al. [19] provided a computational model for the MHD natural convection casson fluid flow with nth order of chemical species and Newtonian heating in porous channel. Their research concentrated on the steady-state one-step exothermic reactions of hydromagnetic flows. However, analysis of steady hydromagnetic double exothermic combustible reaction fluid flow through a porous channel with convective cooling which takes place in a Poiseuille device is yet to be studied. Double exothermic reaction deals with an intermediary reaction. The second exothermic reaction term increases the combustion process. Makinde et al. [20] reported on the heat stability of double steps combustive chemical reaction of a slab. They considered the diffusion reactant by assuming a chemical kinetics of variable factor of the unsteady and steady state. Salawu and Okedoye [21] provided a solution to the unsteady double step exothermic reaction along a conduit but they never considered the case when it's under convective cooling. They studied second law of thermodynamics double step hydromagnetic exothermically combustible chemical reactive fluid flow considering viscous and gravity with heat absorbing along a channel. In the study, they observed that magnetic force decreases the flow rate and undesirable alterations in the fluid viscosity caused by an increase in temperature. Labelo $e t$ al. [22] works on two-step cylindrical stockpiles combustible reaction.

Hence, the purpose of this research is to examine the steady hydromagnetic double reaction of pre-exponential combustible reactive fluid flow in porous channel with convective cooling. However, the important of double combustion reaction processes assist in improving the hydrodynamics lubricants that can leads to engine efficiency. Thus, this study is significant to pollution control, enhancing of industrial machine output and improving lubricant viscosity. An analytical weighted residual technique is employed to provide solutions to the dimensionless viscous dissipative exothermic reaction flow equations. Important of some properties of temperature and velocity field with heat gradient and skin friction are discussed. The present study will help in improving the performance of industrial engines and in reducing toxic discharge to the environment due to incomplete combustion.

This study is motivated by previous report on the important and application on two step exothermic reaction to the chemical industries. Introduction and background to the study is presented in the first section. The problem formulation equations are described in the second section. The collocation weighted residual method of solution is presented and utilized in the third section. The results and discussion is presented in fourth section. The final remark is stated in the last section.

\section{The Flow's Mathematical Formulation}

Consider viscous heating in a reactive fluid of double step combustible reaction between stationary parallel walls. The combustion reaction of the fluid takes place in a Poiseuille device, and it is been propelled by pressure gradient and preexponential bimolecular kinetics. The flow is assumed to be in the direction of $x$ with $y$-axis normal to it as illustrated in Figure 1. The device is exposed to convective cooling to keep the reactive hydromagnetic fluid from distortion. According to Chinyoka and Makinde [23], and ignoring time-dependent and the fluid reactive viscose consumption. The velocity and temperature balance equation governing the flow are as follows:

$$
-\frac{d \bar{p}}{d \bar{x}}+\mu \frac{d^{2} \bar{u}}{d \bar{y}^{2}}-\sigma B_{o}^{2} \bar{u}-\frac{\mu}{k_{1}} \bar{u}=0
$$




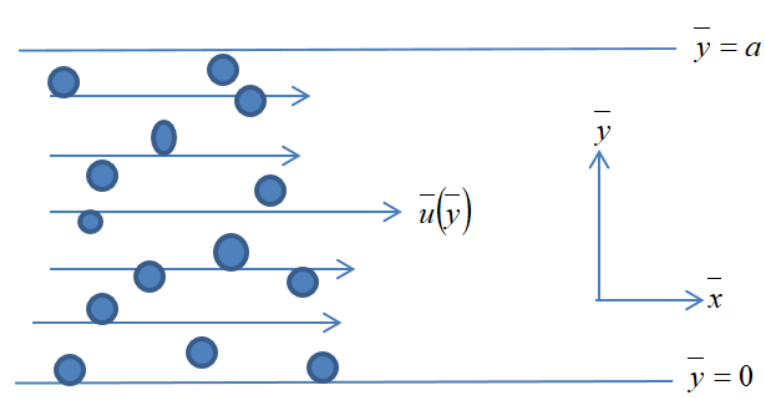

Figure 1: Flow coordinates schematic

$$
\begin{aligned}
& \alpha \frac{d^{2} \bar{T}}{d \bar{y}^{2}}+\mu\left(\frac{d \bar{u}}{d \bar{y}}\right)^{2}+\sigma B_{o}^{2} \bar{u}^{2}+\frac{\mu}{k_{1}} \bar{u}^{2} \\
& \quad+Q_{1} C_{1} A_{1}\left(\frac{K \bar{T}}{v l}\right)^{m} e^{-\frac{E_{1}}{R T}}+Q_{2} C_{2} A_{2}\left(\frac{K \bar{T}}{v l}\right)^{m} e^{-\frac{E_{2}}{R T}}=0
\end{aligned}
$$

The imposing boundary conditions are:

$$
\begin{array}{lll}
\bar{y}=a, & \bar{u}=0, & -k \frac{d \bar{T}}{d \bar{y}}=h\left(\bar{T}-T_{w}\right) \\
\bar{y}=0, & \bar{u}=0, & k \frac{d \bar{T}}{d \bar{y}}=h\left(\bar{T}-T_{w}\right)
\end{array}
$$

Where $B_{0}, \bar{x}, \bar{y}, \bar{p}, \sigma, \bar{u}, \mu, k_{1}, \alpha, Q_{1}, C_{1}, A_{1}, E_{1}, k_{b}, \bar{T}, l, v, R$, $Q_{2}, C_{2}, A_{2}, E_{2}, T_{w}, m$, are magnetic field, dimensional coordinate flow alongside the surface, dimensional coordinate flow along the normal surface, modify pressure, electrical conductivity, axial fluid velocity, fluid viscosity, Darcy porosity, thermal conductivity, first step heat reaction, concentration species of step one reactant, constant rate of step one reactant, energy activation of step one, Boltzmann's constant, dimensional fluid temperature, Plank's number, vibration frequency, general gas constant, step two heat reaction, concentration species of step two reactant, constant rate of step two reactant, energy activation of step two, temperature at the wall, computational index $(m=0.5)$ respectively.

Introducing the following dimensionless quantities:

$$
\begin{aligned}
& \bar{p}=\frac{\mu u p}{a}, \quad \bar{u}=U u, \quad \theta=\frac{E_{1}\left(\bar{T}-T_{w}\right)}{R T_{w}^{2}}, \bar{x}=a x, \\
& H a^{2}=\frac{\sigma B_{o}^{2} a^{2}}{\mu}, G=-\frac{d p}{d x}, y=\frac{\bar{y}}{a}, \\
& B_{r}=\frac{\mu u^{2} E_{1}}{\alpha R T_{w}^{2}}, \quad \lambda=\frac{Q_{1} C_{1} A_{1} a^{2} E_{1}}{\alpha R T_{w}^{2}}\left(\frac{k_{b} T_{w}}{v l}\right)^{m} e^{\frac{1}{\varepsilon}}, \\
& B i=\frac{a h}{k}, H a^{2}=\frac{\sigma B_{o}^{2} U^{2} a^{2}}{\mu}, \\
& K=\frac{a^{2}}{k_{1}}, \quad \gamma=\frac{Q_{2} C_{2} A_{2}}{Q_{1} C_{1} A_{1}} e^{-\frac{1}{\varepsilon}} e^{\frac{1}{\varepsilon}} e^{\frac{r \theta}{(1+\varepsilon \theta)}}
\end{aligned}
$$

Substituting the dimensionless quantities of equation (4) into equation (1) to (2) gives:

$$
\begin{gathered}
\frac{d^{2} u}{d y^{2}}-\left(H a^{2}+K\right) u+G=0 \\
\frac{d^{2} \theta}{d y^{2}}+B r\left[\left(K+H a^{2}\right) u^{2}+\left(\frac{d u}{d y}\right)^{2}\right] \\
+\lambda\left[(1+\varepsilon \theta)^{m}\left(e^{\frac{\theta}{(1+\varepsilon \theta)}}+\gamma e^{\left.\frac{r \theta}{(1+\varepsilon \theta)}\right)}\right)\right]=0
\end{gathered}
$$

alongside with boundaries conditions

$$
u(0)=0, \frac{d \theta}{d y}=-B i \theta, u(1)=0, \frac{d \theta}{d y}=B i \theta
$$

where $\gamma, \lambda, G, B r, H a^{2}, r, \varepsilon$ and $B i$ are two-step exothermic reaction, frank-kamenetskii, pressure gradient, Brinkman numbers, Hartmann numbers, activation energy ratio, activation energy and Biot number parameter respectively.

\section{Numerical setup}

The analytical method of solution used is Weighted Residual Method (WRM). The goal of weighted residual method [24, 25], is to look for an estimated result in the form of polynomial considering the given differential equation.

$$
\Phi[u(v)]=f \text { in the domain } \mathrm{D}, B_{\mu}[u]=\gamma_{\mu} \mathrm{on} \partial D
$$

where $\Phi[u]$ is the non-linear differential operator relating of the dependent variables $\mathrm{u}$ and $\mathrm{f}$ the function of a known point, $B_{\mu}[u]$ is the estimated number of boundary conditions with domain $\mathrm{D}$ and $\partial D$ the boundary. The solution to the problem of the above estimated boundary isoften carried out by taking an estimate of the solution $u(v)$. The expression

$$
u(v) \approx w\left(v, a_{1}, a_{2}, \ldots a_{t}\right)
$$

relies on the number of parameters $a_{1}, a_{2}, \ldots . . a_{t}$ and the arbitrary value $a_{j}^{l}$ of the boundary conditions are satisfied. In practice $w\left(v, a_{1}, a_{2}, \ldots a_{t}\right)$ take the form

$$
w(v, a)=\varphi_{o}(v)+\sum_{j=1}^{t} a_{j} \varphi_{j}
$$

where the function $\varphi_{j}(v)$ are given to satisfy the boundary conditions

$$
Q_{\mu}\left(\varphi_{o}\right)=\gamma_{\mu}, Q_{\mu}\left(\varphi_{j}\right)=0, j=1,2.3 . . . t
$$

For arbitrary values of $a^{l} s, w\left(v, a_{1}, a_{2}, a_{3}, \ldots a_{t}\right)$ fulfills the boundary condition (8).

When equation ( 9 is substituted for equation (8), the differential equation's residual becomes

$$
\phi(v, a)=L\left(w\left(v, a_{i}\right)\right)-f(v)
$$

where $a=\left(a_{1}, a_{2}, \ldots . a_{t}\right)$.This yields the measure whereby the function $w(v, a)$ satisfies the differential equation's degree, as the number $t$ of the function ' $j$ is enhanced in subsequent estimations. The aim is to minimize the residual $\phi(v, a)$ to zero on an average basis throughout the domain. Which is

$$
\int_{c} \phi(v, a) W_{j} d v=0, \quad j=1,2, \ldots t
$$


where the unknown number of constants $a_{j}$ in $w$ is the same as the value of weight functions $W_{j}$. The outcome consist an algebraic equations of set $t$ for all the unidentifiedconstants $a_{j}$. So, functions of the weighted are taken as Dirac delta. That is, $W_{j}(v)=\delta\left(v-v_{j}\right)$ such that the chosen error at the node $v_{j}$ is zero. Integrating equation (13) with $W_{j}(v)=\delta\left(v-v_{j}\right)$ result in $\phi\left(v, a_{j}\right)=0$. Employing WRM to equations (5), (6) and (7), assuming that the parameters or polynomial with undefined coefficients will be found later, this function is referred to as the trial function.

$$
u(v)=\sum_{j=0}^{10} a_{j} v^{j}, \theta(v)=\sum_{j=0}^{10} b_{j} v^{j}
$$

Introducing the boundary condition (7) on (14) which is the trial functions and then substitute the trial function on the equation (5) and (6) to get the residual of the momentum and energy equation as:

$$
\begin{aligned}
& u_{r}=90 y^{8} a_{10}+72 y^{7} a_{9}+56 y^{6} a_{8}+42 y^{5} a_{7}+30 y^{4} a_{6}+20 y^{3} a_{5}+12 y^{2} a_{4}+ \\
& 6 y a_{3}+2 a_{2}-\left(H a^{2}+K\right)\left(\begin{array}{l}
y^{10} a_{10}+y^{9} a_{9}+y^{8} a_{8}+y^{7} a_{7}+y^{6} a_{6}+y^{5} a_{5}+ \\
y^{4} a_{4}+y^{3} a_{3}+y^{2} a_{2}+y a_{1}+a_{0}
\end{array}\right)+G \\
& \theta_{r}=90 y^{8} b_{10}+72 y^{7} b_{9}+56 y^{6} b_{8}+42 y^{5} b_{7}+30 y^{4} b_{6}+20 y^{3} b_{5}+12 y^{2} b_{4}+6 y b_{3}+2 b_{2}+
\end{aligned}
$$

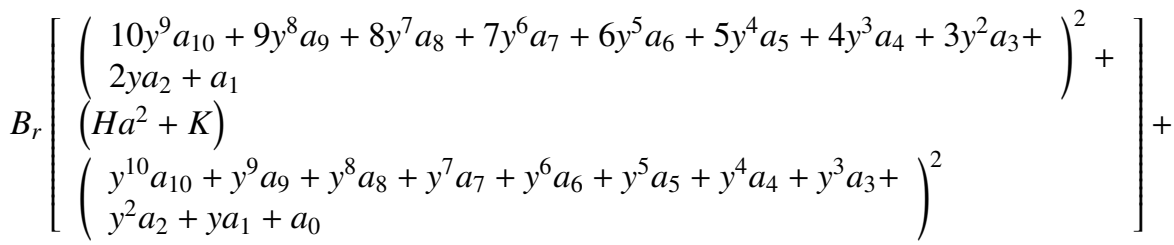

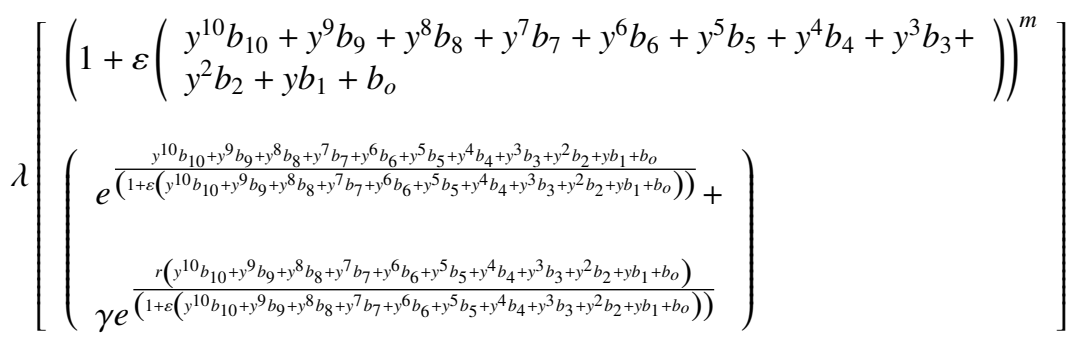

The residual for the set of collocation points within the domain at regular interval is reduced to zero when $m=0.5, B r=1, \varepsilon=$ $1, \gamma=1, \lambda=0.5, G=1, H a=1, K=1$.

That is $N_{n}=\frac{(q-p) n}{M}$ where $n=1,2,3, . ., M-1$ and $p=0, q=1, M=9$. The unknown constant coefficients are gotten with the aid of MAPLE software. So, the dimensionless equations are as follow:

$$
\begin{aligned}
& u(y)=-0.000003521116925 y^{10}+0.00001760557764 y^{9}-0.0001970055654 y^{8}+ \\
& 0.0006823888193 y^{7}-0.005555067018 y^{6}+0.01435078358 y^{5}- \\
& 0.08333329281 y^{4}+0.1435095221 y^{3}-0.49999999994 y^{2}+0.4305285858 y \\
& \theta(y)= 0.000317754724 y^{10}-0.001588772547 y^{9}+0.007075226439 y^{8}- \\
& 0.01876827406 y^{7}+0.05145794359 y^{6}-0.09535771165 y^{5}+ \\
& 0.1277972545 y^{4}-0.1153837668 y^{3}+0.2601529854 y^{2}- \\
& 0.2157026397 y+0.2157026397
\end{aligned}
$$

The process of WRM scheme is used for difference value of. $K, \lambda, H a^{2}, G, \gamma, \varepsilon$ and $B r$. The physical quantities that are of interest to engineering are the heat gradient $(\mathrm{Nu})$ and skin friction $\left(C_{f}\right)$ respectively as $[23,27,28]$.

$$
N u=-\frac{d \theta}{d y} \quad \text { and } \quad C_{f}=\frac{d u}{d y}
$$




\section{Results and Discussion}

The numerical result in Table 1 depicts the influence of many physical factors on skin friction and nusselt numbers. A rise in values of the term $\mathrm{G}$ and $\lambda$ accelerate the temperature gradient and skin friction at the wall since temperature at the boundary layer increases. Also, an increase in values of $\mathrm{Ha}$ and $\mathrm{K}$ decreases the effect flow at the wall surface. Table 2 displays the relationship between the existing related work and the current results. Thus, the current results are consistent with the previous results. Table 3 demonstrates the comparison results of the exact and the existing work. The outcome shows that they are in good agreement.Figure 2 demonstrates the profile of velocity over pressure gradient $(G)$. An increase in $(G)$ term enhances the fluid flow in the channel and heat within the system rises which cause the breaking of the force bonding in the fluid. Hence, pressure gradient aids changes in formation of fluid in the system. Figure 3 shows effect of fluid velocity on Hartmann number $(\mathrm{Ha})$. The Lorentz force present in $(\mathrm{Ha})$ increases the damping magnetic strength which then accelerate the fluid resistance and by that decelerate the motion of the fluid on convective cooling wall. Thus, the velocity profile is affected by a very small reading scale system which helps in fluid viscosity of many industries. Figure 4 demonstrates porosity parameter $(\mathrm{K})$ which generates resistance force in the fluid as shown in the plot. Increase in porosity $(K)$ values lead to an increase of the resistance in the fluid. This is due to the saturated of the medium with pores that decreases the free flow of the fluid. The term enhances viscosity fluid as a result of decrease within the heat source terms and so reduces the velocity flow.

Figure 5 displayed the result of Frank-kamenetskii parameter $(\lambda)$. A rise in $(\lambda)$ correspond to an increase in the rate of viscous heating in a reaction which enhanced the chemically reaction rate and consequently increase the distribution of the temperature within the system. This is due to enhancement in the momentum viscosity coupling that results in rational rises in the flow temperature. The parameter $(\lambda)$ can cause a blow up if not well monitored. Figure 6 shows the strength of activation energy $(\varepsilon)$ on heat transfer in an exothermic chemical reactive system. The least energy which exists in a chemical system with potential reactants is known as activation energy. As the parameter term $(\varepsilon)$ rises the level of heat transfer decreases. This is because of the presence of an appreciable number of molecules with translational energy equal to or greater than the activation energy. Thus, this help the industries to inspect the spark of activation energy required to break the bond in a chemical reaction. Figure 7 reveals that temperature falls while the porosity $(\mathrm{K})$ values rises because the resistance to fluid motion offered by porous medium causes the fluid to heat up, so the temperature decreases within the boundary layer. The behavior is as a result of the wall of the plates that provides a supporting resistance to the fluid flow. Figure 8 presents the influence of second step term $(\gamma)$ on the temperature field. It reveals that the double step exothermic combustive reaction increases heat transfer in the system. Thus, this leads to increase in the combustion process that in turn encourages complete burn of hydrocarbon in the engines. Hence, second step exothermic reaction

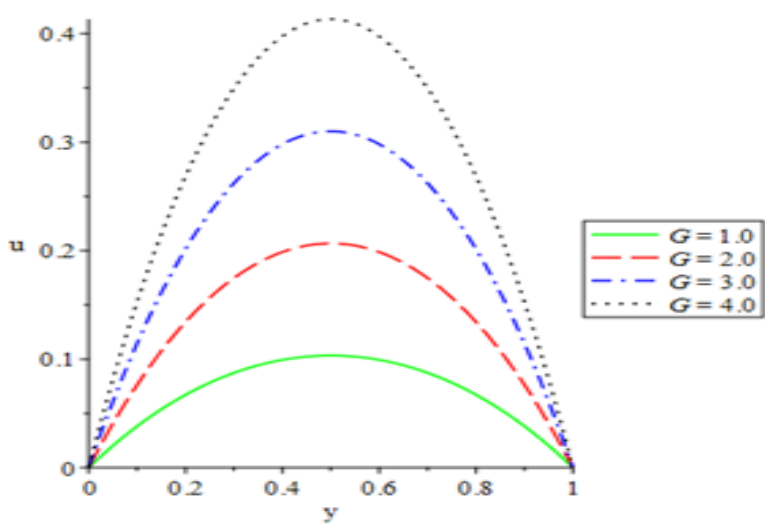

Figure 2: Profile of velocity against $(G)$

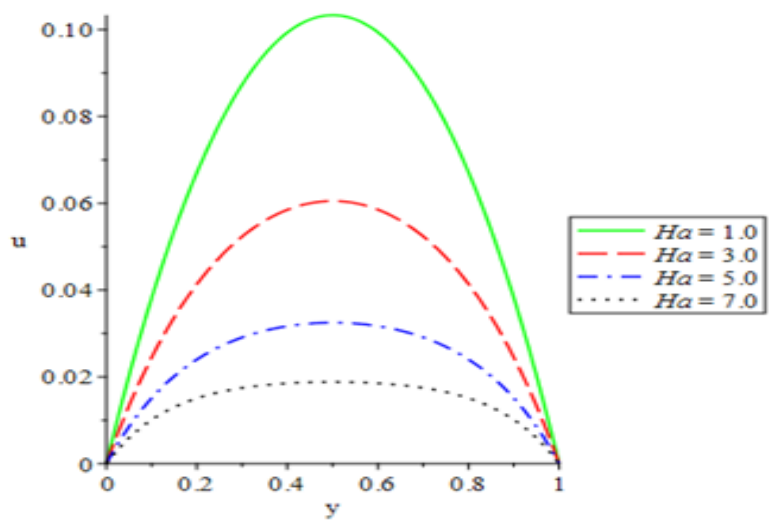

Figure 3: Profile of velocity against $(\mathrm{Ha})$

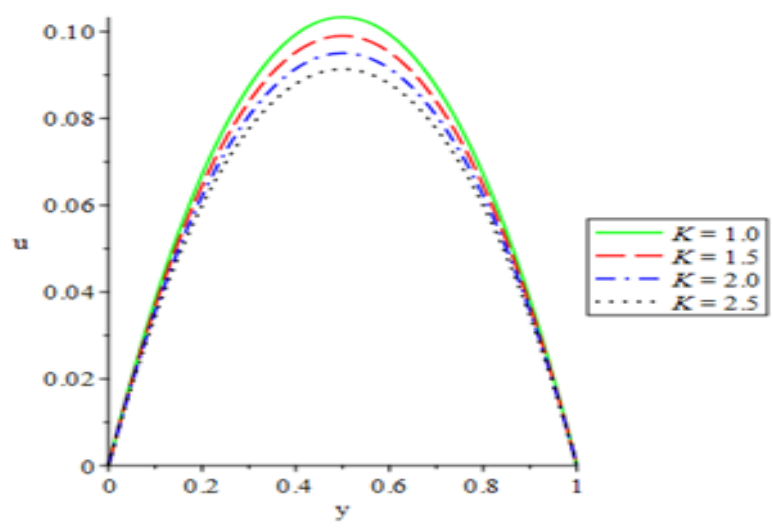

Figure 4: Profile of heat against $(K)$

reduces the release of carbon-monoxide that pollutes our environment as a result of unburned hydrocarbon. 
Table 1: Comparison of $C_{f}$ and $N u$ at various values of $G, H a^{2}, K$ and $\lambda$

\begin{tabular}{cccccccc}
\hline Parameter & Values & \multicolumn{2}{l}{$\begin{array}{l}\text { Weighted Resid- } \\
\text { ual method }\end{array}$} & \multicolumn{2}{l}{$4^{\text {th }}$ order of R-K } & \multicolumn{2}{l}{ Absolute error } \\
& \multicolumn{2}{c}{$C_{f}$} & $N u$ & $C_{f}$ & $N u$ & $C_{f}$ & $N u$ \\
\hline$G$ & 1.0 & 0.430529 & 0.290443 & 0.430529 & 0.290443 & $7.0 \times 10^{-8}$ & $1.4 \times 10^{-8}$ \\
\hline & 2.0 & 0.861057 & 0.344304 & 0.861057 & 0.344304 & $1.7 \times 10^{-9}$ & $1.2 \times 10^{-7}$ \\
\hline & 3.0 & 1.291586 & 0.434728 & 1.291586 & 0.434729 & $3.0 \times 10^{-9}$ & $7.3 \times 10^{-7}$ \\
\hline$H a^{2}$ & 4.0 & 1.722114 & 0.564353 & 1.722114 & 0.564357 & $1.8 \times 10^{-9}$ & $4.0 \times 10^{-6}$ \\
\hline & 1.0 & 0.430529 & 0.290443 & 0.430529 & 0.290443 & $8.0 \times 10^{-10}$ & $1.4 \times 10^{-8}$ \\
\hline & 3.0 & 0.290544 & 0.283715 & 0.290544 & 0.283717 & $5.1 \times 10^{-8}$ & $1.1 \times 10^{-6}$ \\
\hline & 5.0 & 0.193735 & 0.278940 & 0.193737 & 0.278951 & $2.4 \times 10^{-6}$ & $1.0 \times 10^{-5}$ \\
\hline$K$ & 7.0 & 0.141156 & 0.276443 & 0.141181 & 0.276472 & $2.5 \times 10^{-5}$ & $2.9 \times 10^{-5}$ \\
\hline & 1.0 & 0.430529 & 0.290443 & 0.430529 & 0.290443 & $2.8 \times 10^{-8}$ & $1.4 \times 10^{-8}$ \\
\hline & 1.5 & 0.416618 & 0.289792 & 0.416618 & 0.289792 & $1.4 \times 10^{-9}$ & $2.1 \times 10^{-8}$ \\
\hline$\lambda$ & 2.0 & 0.403769 & 0.289188 & 0.403769 & 0.289187 & $1.9 \times 10^{-9}$ & $3.3 \times 10^{-8}$ \\
\hline & 2.5 & 0.391863 & 0.288624 & 0.391863 & 0.288624 & $2.6 \times 10^{-9}$ & $5.0 \times 10^{-8}$ \\
\hline & 1.0 & 0.430529 & 0.388391 & 0.430529 & 0.388391 & $8.0 \times 10^{-10}$ & $1.7 \times 10^{-8}$ \\
\hline & 3.0 & 0.430529 & 0.526687 & 0.430529 & 0.526686 & $2.0 \times 10^{-10}$ & $3.7 \times 10^{-7}$ \\
\hline & 5.0 & 0.430529 & 0.599584 & 0.430529 & 0.599524 & $1.0 \times 10^{-10}$ & $6.0 \times 10^{-5}$ \\
\hline & 7.0 & 0.430529 & 0.703732 & 0.430529 & 0.707863 & $1.0 \times 10^{-10}$ & $4.1 \times 10^{-3}$ \\
\hline
\end{tabular}

Table 2: Comparison of temperature profile for $\mathrm{G}=\lambda=1, \varepsilon=\mathrm{Ha}=\mathrm{Br}=1$

\begin{tabular}{cccc}
\hline $\mathrm{y}$ & Makinde and Beg [28] & Hassan and Maritz [13] & Present study \\
\hline 0.00 & 0.3544502181 & 0.3581076494 & 0.3582159913 \\
\hline 0.25 & 0.3323243479 & 0.3358417798 & 0.3358879051 \\
\hline 0.50 & 0.2660663845 & 0.2691056991 & 0.2690827375 \\
\hline 0.75 & 0.1556934861 & 0.1577379743 & 0.1578252719 \\
\hline 1.00 & 0 & 0.0002178316 & 0.0000182633 \\
\hline
\end{tabular}

Table 3: Comparison of the exact results with WRM for $\mathrm{u}(\mathrm{y})$ when $\mathrm{G}=\mathrm{K}=\mathrm{Ha}=1$

\begin{tabular}{ccc}
\hline $\mathrm{y}$ & Exact solution for $\mathrm{u}(\mathrm{y})$ & Weighted residual method for $\mathrm{u}(\mathrm{y})$ \\
\hline 0.0 & 0 & 0 \\
\hline 0.2 & 0.0671247049 & 0.0671247051 \\
\hline 0.4 & 0.0993879030 & 0.0993879032 \\
\hline 0.6 & 0.0993879029 & 0.0993879032 \\
\hline 0.8 & 0.0671247053 & 0.0671247050 \\
\hline 1.0 & 0.0000000001 & 0 \\
\hline
\end{tabular}




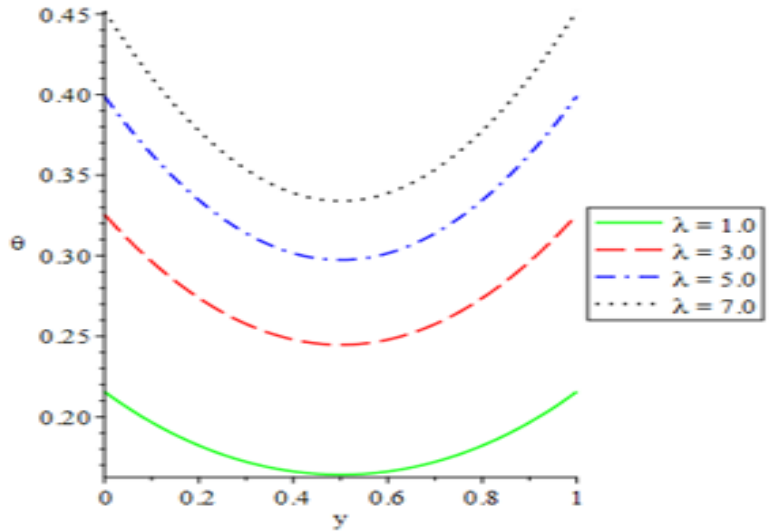

Figure 5: Profile of heat against $(\lambda)$

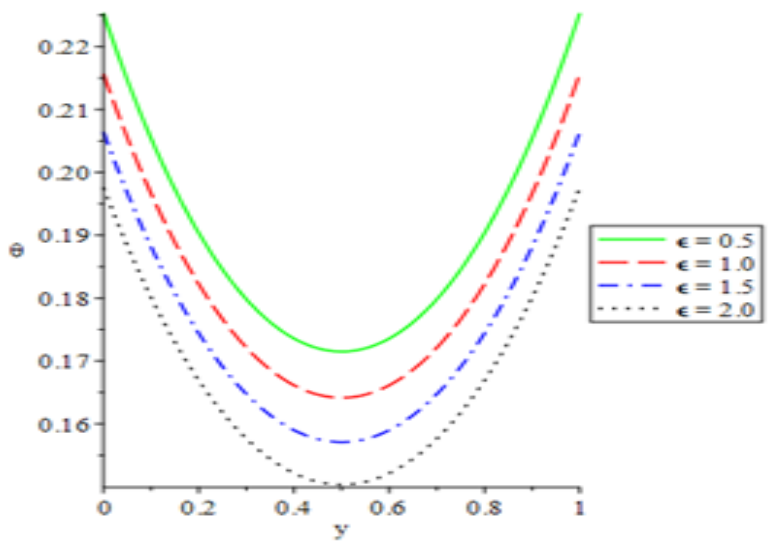

Figure 6: Profile of heat against $(\varepsilon)$

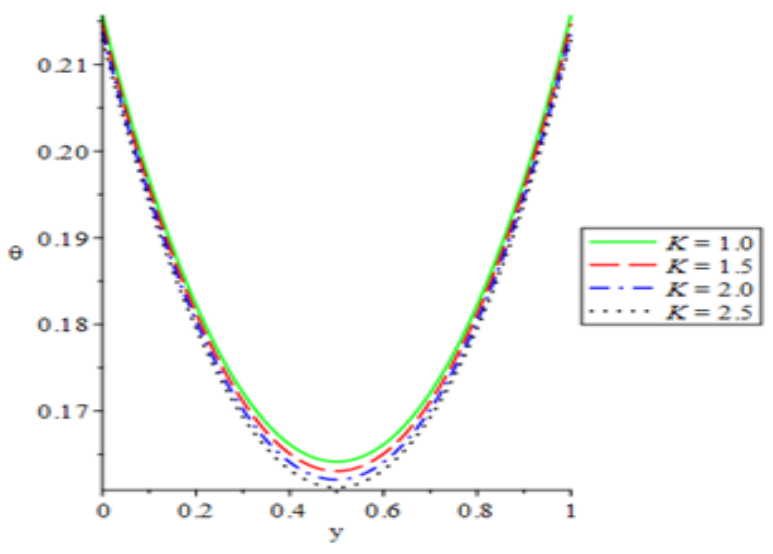

Figure 7: Profile of heat against $(K)$

\section{Conclusion}

The analysis of steady hydromagnetic double exothermic chemically reactive flow with convective cooling through a porous medium under bimolecular kinetics was mathematically formulated. The weighted residual collocation integration method were used to solve the dimensionless viscous flow equations and the computational result obtained were used to compare with the fourth order of Runge-schemes coupled by shooting

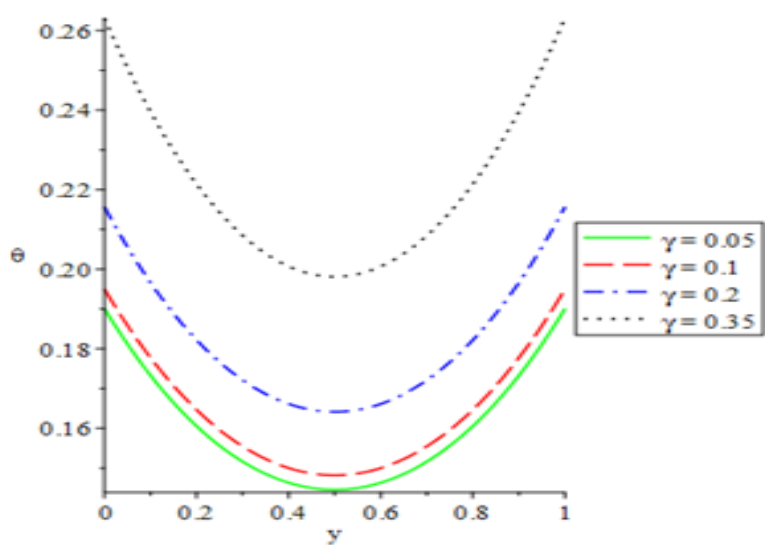

Figure 8: Profile of heat against $(\gamma)$

techniques, and found to have very low absolute errors. As obtained in the study, the second exothermic reaction term increases the combustion of hydrocarbon due to rise in the heat distribution within the system. Hence the term will assist in reducing toxic discharge from the engines that pollute the environment. Also, terms that increase heat in the system must be guarded to prevent solution explosion. This work is significant in improving combustion process in the engines with many industrial applications. Hence, this study is limited to Newtonian fluid and could be extended to a non-Newtonian flow in an annular cylindrical pipe.

\section{References}

[1] R. Ellahi \& S. Afzal, "Effects of variable viscosity in a third grade fluid with porous medium:an analytic solution", Communications in Nonlinear Science and Numerical Simulation 14 (2009) 2056.

[2] O. D. Makinde, "Similarity solution for natural convection from a moving vertical plate with internal heat generation and a convective boundary condition", Thermal Science 15 (2011) S137.

[3] C. Chen, "On analytic solution of MHD flow and heat transfer for two types of viscoelastic fluid over a stretching sheet with energy dissipation, internal heat source and thermal radiation", International Journal of Heat Mass Transfer 53 (2010) 4264.

[4] F. Mabood \& S. M. Ibrahim, "Effects of soret and non-uniform heat source on MHD non-Darcian convective flow over a stretching sheet in a dissipative micropolar fluid with radiation", Journal of Applied Fluid Mechanics 9 (2016) 2503.

[5] F. Mabood, S.M. Ibrahim, M. M. Rashidi, M. S. Shadloo \& L. Giulio, "Non-uniform heat source/sink and Soret effects on MHD non-Darcian convective flow past a stretching sheet in a micropolar fluid with radiation", International Journal of Heat and Mass Transfer 93 (2016) 674.

[6] J. A. Gbadeyan \& A. R. Hassan, "Multiplicity of solutions for a reactive variable viscous Couette flow under Arrhenius kinetics", Math Theory Model 2 (2012) 39.

[7] V. D i Marcello, L. Cammi \& A. Luzzi. "Generalized approach to heat transfer in pipe flow with internal heat generation", ChemEngSci $\mathbf{6 5}$ (2010) 1301

[8] M. F. El-Amin, "Combined effect of internal heat generation and magnetic field on free convection and mass transfer flow in a micro polar fluid with constant suction", J Magn Mater 270 (2004) 130.

[9] A. R . Hassan \& J. A. Gbadeyan, "A reactive hydromagnetic internal heat generating fluid flow through a channel", Int. J. Heat and Technol. 33 (2015) 43.

[10] G. C. Hazarika, K. Goswami \& J. Konch, "Effects of variable viscosity and thermal conductivity on MHD flow past a vertically moving porous 
plate with viscous and joule dissipation", International Journal of Computer Applications 123 (2015) 40.

[11] P. Dulal \& M. Hiranmoy, "Effects of temperature-dependent viscosity and variable thermal conductivity on MHD non-Darcy mixed convective diffusion of species over a stretching sheet", Journal of the Egyptian Mathematical Society 22 (2014) 123.

[12] D. Hunegnaw \& K. Naikoti, " MHD effects on heat transfer over stretching sheet embedded in porous medium with variable viscosity, viscous dissipation and heat source/sink", Ain Shams Engineering Journal 5 (2014) 967.

[13] A. R. Hassan \& R. Maritz "The analysis of a reactive hydromagnetic internal heat generation poiseuille fluid flow through a channel", SpringerPlus 5 (2016) 132

[14] P. Dulal "Heat and mass transfer in stagnation-point flow towards a stretching surface in the presence of buoyancy force and thermal radiation", Meccanica 44 (2009) 145.

[15] G. C. Hazarika \& S. G. Ch. Utpal, "Effects of variable viscosity and thermal conductivity on MHD flow past a vertical plate", Matematicas Ensenanza Universitaria 2 (2012) 45.

[16] S. O. Adesanya \& J A. Folade, "Thermodynamics analysis of hydromagnetic third grade fluid flow through a channel filled with porous medium". Alexandria Eng. J. 54 (2015) 615.

[17] P. A. Bala \& S. Suneetha, "Aspects of homogeneous-heterogeneous chemical reaction and slip velocity on MHD stagnation flow of a micropolar fluid over a permeable stretching/shrinking surface embedded in a porous medium", (2017) 837.

[18] S. O. Salawu, "Analysis of third-grade heat absorption hydromagnetic exothermic chemical reactive flow in a Darcy-Forchheimer porous medium with convective cooling", WSEAS Trans. Math. 17 (2018) 280.

[19] G. S. Seth, A. Bhattacharyya, R. Kumar \& M. K. Manoj, "Modelling and numerical simulation of hydromagnetic natural convection casson fluid flow with nth order chemical reaction and Newtonian heating in porous medium", Journal of Porous Media 22 (2019) 1141.

[20] O. D. Makinde, P. O. Olanrewaju, E. O. Titiloye, \& A.W. Ogunsola, "On thermal stability of a two-step exothermic chemical reaction in a slab", Journal of Mathematics science 13 (2013) 1.

[21] S. O. Salawu \& A.M. Okedoye, "Thermodynamic second law analysis of hydromagnetic gravity-driven two-step exothermic chemical reactive flow with heat absorption along a channel", Iranian Journal of Energy and Environment 9 (2018) 114.

[22] R. S Lebelo, R. K. Mahlobo \& K. C. Moloi, "Thermal stability analysis in a two-step reactive cylindrical stockpile", American Journal of Applied Sciences 15 (2018) 124.

[23] T. Chinyoka \& O. D. Makinde, "Analysis of transient Generalized Couette flow of a reactive variable viscosity third-grade liquid with asymmetric convective cooling", Mathematical and Computer Modelling 54 (2011) 160.

[24] S. O. Salawu, R. A. Oderinu, \& A. D Ohaegbue, "Thermal runaway and thermodynamic second law of a reactive couple stress fluid with variable properties and Naviier slips", Scientific African 7 (2020) 1

[25] S. A. Odejide \& Y. A. S. Aregbesola, "Application of weighted residuals to problems with semi-finite domain.” Rom. Journ. Phys. 56 (2011) 14.

[26] S. O. Salawu \& A. B. Disu, "Branched-chain criticality and explosion for a generalized thermal Oldroyd 6-constant coquette reactive fluid flow", South African Journal of Chemical Engineering 34 (2020) 90.

[27] S. O. Salawu \& S. S. Okoya, "On criticality for a branched-chain thermal reactive-diffusion in a cylinder", Combustion Science and Technology 192 (2020) 1.

[28] O. D. Makinde \& O. A. Beg, "On inherent irreversibility in a reactive hydromagnetic channel flow." J. Them. Sci. 19 (2010) 72. 